\title{
Common Pedagogical Issues with De Broglie Waves: Moving Double Slits, Composite Mass, and Clock Synchronization
}

\author{
Robert L. Shuler \\ NASA Johnson Space Center, Houston, TX 77058, USA \\ Correspondence should be addressed to Robert L. Shuler; mclsoft@yahoo.com
}

Received 28 September 2015; Accepted 9 November 2015

Academic Editor: Martin Kröger

Copyright ( 2015 Robert L. Shuler. This is an open access article distributed under the Creative Commons Attribution License, which permits unrestricted use, distribution, and reproduction in any medium, provided the original work is properly cited.

\begin{abstract}
This paper addresses gaps identified in pedagogical studies of how misunderstanding of De Broglie waves affects later coursework and presents a heuristic for understanding the De Broglie frequency of composite. De Broglie's little known derivation is reviewed with a new illustration based on his description. Simple techniques for reference frame independent analysis of a moving double slit electron interference experiment are not previously found in any literature and cement the concepts. Points of similarity and difference between De Broglie and Schrödinger waves are explained. The necessity of momentum, energy, and wavelength changes in the electrons in order for them to be vertically displaced in their own reference frame is shown to be required to make the double slit analysis work. A relativistic kinematic analysis of De Broglie frequency is provided showing how the higher De Broglie frequency of moving particles is consistent with Special Relativity and time dilation and that it demonstrates a natural system which obeys Einstein's clock synchronization convention of simultaneity and no other. Students will be better prepared to identify practical approaches to solving problems and to think about fundamental questions.
\end{abstract}

\section{Introduction}

At some point the undergraduate physics student and many engineering or chemistry students as well will take a one semester course in quantum mechanics (QM). When the student encounters things like De Broglie wavelengths and double slit experiments, no mention is made of how to analyze the problem from any other reference frame, not even with a simple Galilean transformation relationship. Yet any student that actually looks at and thinks about $\lambda=h / \rho$ will feel puzzled. How can a wavelength that varies in that way with momentum and thus velocity be a real physical thing? How could one analyze it from another reference frame? The wavelength has an inverse dependence on velocity and becomes infinite in the particle's own reference frame.

Studies of the wave-particle knowledge of university students completing a modern physics course suggest that only the most insightful students will get as far as suggested above, to become puzzled. Many bring incorrect ideas from misunderstanding optics to bear on electrons, such as thinking photons "bend at the slit edges" in diffraction, and compound their misunderstanding by "failure to recognize the De Broglie wave is not an inherent property of an electron but varies with momentum," thinking that "diffraction and interference are independent of velocity," and believing that "equations that apply to the wavelength of light apply to De Broglie wavelength" [1].

An experienced instructor doubtless will feel that, in an already crowded syllabus, it is best to solve each problem in the simplest reference frame. For the case of electron interference or diffraction, this is the frame of the slit(s). But the instructor is already clear on the concepts and with a junior or senior class by now skilled in memorizing formulae and passing exams, the misconceptions from earlier courses may persist. This paper attempts to show that, in the case of De Broglie wavelength, a short excursion into another reference frame may be precisely the exercise that will allow students to identify and correct misconceptions quickly and effectively.

Actually De Broglie struggled with relativity while developing his theory [2]. But De Broglie started with frequency and the idea of a nonpropagating wave that had the same phase everywhere. The problem was that this was incompatible with Special Relativity, and to fix the problem 


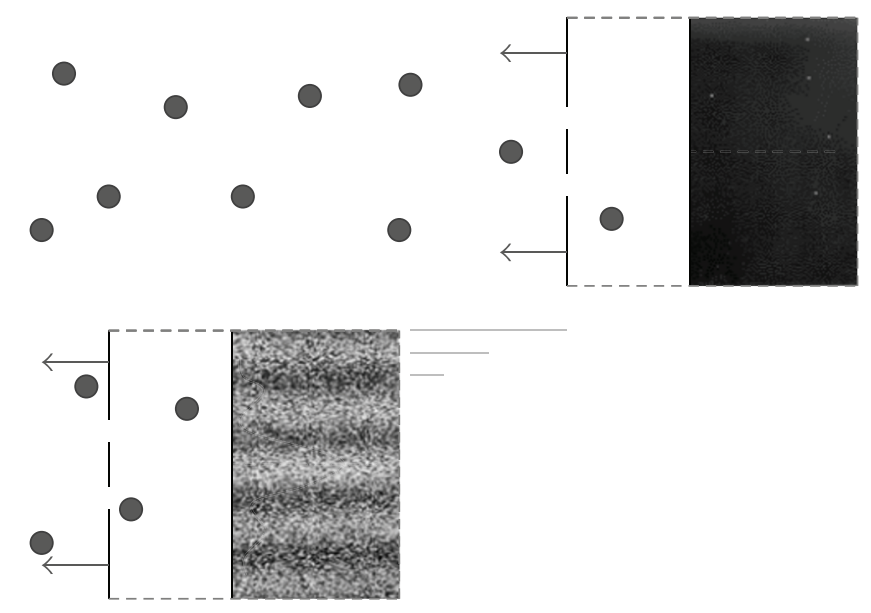

FIGURE 1: Double slit moving through electron cloud.

he applied the Lorentz transform and found that, in frames other than the particle's frame, a phase wave and associated wavelength appear as a result. It is similar in some ways to the idea that the magnetic field is due to the effects of relativity on the electrostatic field, which has been known for more than a century [3] and taught to undergraduates for nearly half a century, popularized in Purcell's 1963 textbook [4].

For a simple example of the puzzle of applying De Broglie waves in certain reference frames, consider a double slit experiment from the point of view of the electrons as shown in Figure 1. The resulting pattern will be the same regardless of who is moving. But how can this be if the wavelength becomes infinite?

In the whirlwind of new information and possibly persistent misconceptions from prior courses, students may not even verbalize the puzzle and if they do, instructors may find themselves surprised and unsure how to answer. In this way a vague sense of mystery is unnecessarily passed to the next generation, and occasionally discussions about how to apply the Lorentz transform to a De Broglie wavelength crop up on physics discussion forums [5]. Such discussions never mention that De Broglie derived the wavelength as an artifact of the Lorentz transformation of a wave of simultaneity, resulting in the desynchronization of clocks seen by relatively moving observers, because this historical derivation is not one of the minimal sets captured by and repeated in QM textbooks, most of which seem to follow very similar outlines. In fact there are no less than three recent papers claiming to have "discovered" that De Broglie waves were such an artifact of the Lorentz transform (LT) [6-8].

The correct thing to transform is of course momentum, or the particle's momentum vector, not the wavelength, which is simply recomputed after transformation of the momentum vector. Or one can use the wave 4 -vector which is related to momentum $\mathbf{k}=\boldsymbol{\rho} / \hbar$. This works fine until the velocities become low, and finally in the rest frame of the particles $\mathbf{k}=\boldsymbol{\rho}=0$ for which interference is impossible as the wave is in-phase everywhere, and one is faced with solving scattering problems to recover a situation where there are finite wavelengths, problems which did not need to be considered in other reference frames. (Note that De Broglie wavelength which causes interference and diffraction among like particles, or a particle itself, is distinct from the Compton wavelength which is never zero and explains photon scattering from particles.)

For the benefit of those who may go on to study relativity, it will be demonstrated that since the wavelength is dependent on the LT, the interference pattern is dependent on the underlying clock synchronization convention suggested by Einstein and confirms it as a property of a natural system.

A modern student is additionally confronted with De Broglie-style interference not only for electrons [9], but even for multiparticle objects, such as $\mathrm{C}_{60}$ [10]. This can bring up questions such as how the individual particle frequencies "add up," or even whether the De Broglie wave is a particle or a mass concept [11]. The limited theoretical work on this is analyzed and a simple heuristic developed which provides a satisfactory answer to first order.

\section{Origin of the De Broglie Wavelength}

De Broglie recounts his thought process in his 1929 Nobel Lecture [12]:

\begin{abstract}
In the "intrinsic" system of the corpuscle in the sense of the relativity theory... the wave will be stationary since the corpuscle is immobile: its phase will be the same at every point. (emphasis added)
\end{abstract}

An illustration of how the De Broglie wavelength arises from the Lorentz transformation is shown in Figures 2(a) and 2(b). A point of constant phase on the De Broglie wave is a point of simultaneity in the reference frame of the particle. This is shown by a row of synchronized clocks in Figure 2(a), in which time $t$ is the vertical axis and one of the spatial dimensions, $x$, is the horizontal axis. This wave is propagating in time but not in space. It represents the simultaneous probability wave of the particle in its rest frame. It may have a spatial shape representing different probabilities in different spatial regions, but the phase points of this shape will not be moving. Dashed lines show points of constant phase and therefore constant time. Three clocks also in the rest frame of the particle emphasize the constant time of a particular phase.

In Figure 2(b), the particle and associated wave and reference frame clocks are viewed by an observer traveling to the left. The illustration is in the frame of the observer so the clocks of the particle frame appear skewed with leading clocks lagging as shown. This means that some particular phase point has not yet arrived at the leading clocks (on the right) but has already passed clocks on the left which are later in time. We will derive the De Broglie wavelength from this illustration in the next section. 


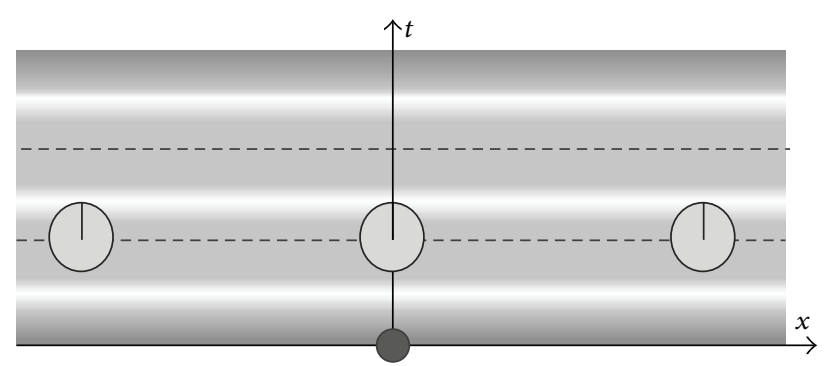

(a)

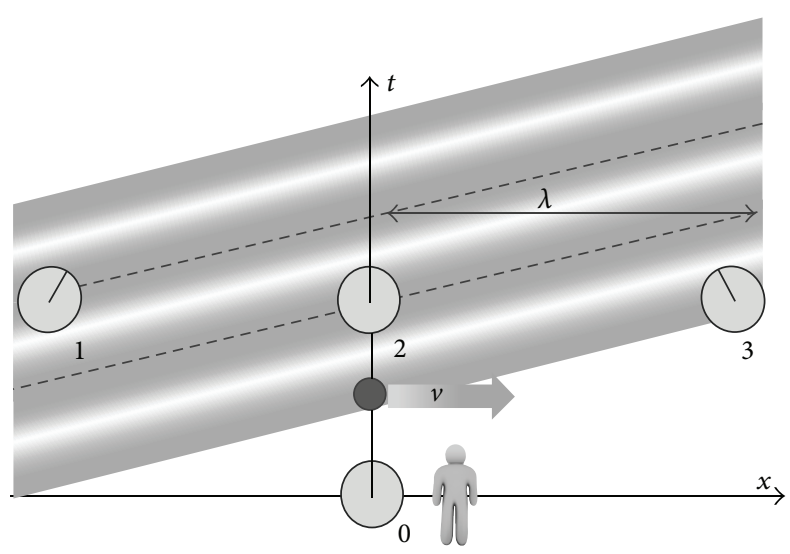

(b)

Figure 2: (a) De Broglie waves in rest frame of particle. (b) De Broglie waves seen from another inertial frame.

\section{Derivation of De Broglie Wavelength and Phase Velocity}

De Broglie first postulated a vibration associated with particles and used the quantum mechanical formulation recently discovered by Planck to assert [13]

$$
E=h f \quad\left(\text { alternately } f=\frac{E}{h} \text { or } f=\frac{\gamma m c^{2}}{h}\right) .
$$

$E$ is the total relativistic particle energy, $h$ is Planck's constant, $f$ is frequency, and $\lambda$ is the Lorentz gamma factor $(1-$ $\left.v^{2} / c^{2}\right)^{-1 / 2}$. Some textbooks mention this in one sentence and then proceed with a much longer discussion of wavelength, without any derivation, as the more important focus for understanding and solving problems [14].

To obtain the De Broglie wavelength, we calculate $\lambda$ in Figure 2(b) as the Lorentz clock skew matching the oscillation period $1 / f$ as follows:

Use Lorentz coordinate time transformation: $t^{\prime}=$ $\gamma\left(t-v x / c^{2}\right)$.

Solve for time skew equal to the period $1 / f=t^{\prime}-t$.

Use $t=0$ without losing generality.

Find $x^{\prime}$ which gives $1 / f=t^{\prime}$ using time transformation $\Rightarrow 1 / f=\gamma\left(v x / c^{2}\right) \Rightarrow x=c^{2} / f v \gamma$.

Using (1) we have $x=h c^{2} / \gamma m c^{2} \gamma v=h / \gamma m \gamma v$.

Since $x$ is in the unprimed (particle) frame, use coordinate transformation: $x^{\prime}=\gamma(x-v t)$.

For $t=0, x^{\prime}=\gamma x=h / \gamma m v=h / \rho$.

Since $x^{\prime}$ is the distance between adjacent points of constant phase $x^{\prime}=\lambda$, we have

$$
\lambda=\frac{h}{\rho} .
$$

De Broglie then associated this wavelength with a superluminal wave with phase velocity propagating at $v_{\text {ph }}$ greater than the velocity of light. The author would prefer not to use the terminology "superluminal wave" since in the reference frame of the particle the wave is not propagating at all. Points of constant phase are only appearing to move due to Lorentz clock skew, and we can easily calculate this apparent velocity as the ratio of the $x^{\prime}$ distance we just derived $(\lambda)$ to the period $1 / f$ and find that

$$
v_{\mathrm{ph}}=\lambda f=\frac{h E}{h \rho}=\frac{E}{\rho}=\frac{c^{2}}{v} .
$$

This superluminal velocity nonetheless bothered De Broglie very much. Based on work done by Marcel Brillouin, father of De Broglie's girlfriend, he solved this problem by creating a wave packet in which the group velocity $v_{\mathrm{gr}}$ corresponds to the particle velocity $v$, and substituting $v_{\text {gr }}$ for $v$ in (3) immediately gives the relation:

$$
v_{\mathrm{gr}} v_{\mathrm{ph}}=c^{2} \text {. }
$$

Introductory QM textbooks for one semester courses may immediately proceed to derive a plane wave example for which $v_{\mathrm{ph}}=v_{\mathrm{gr}} / 2$ and that may be the last quantitative mention of phase velocity. Probably this is because authors feel it is more important to move students quickly into the Schrödinger equation. This is not the group-phase velocity relation for a true De Broglie wave. It is the correct phase velocity for a solution to the Schrödinger equation. It may be mentioned later in a text that phase velocity is an artifact of the choice of energy zero point and not otherwise meaningful, but in the meantime confusion may reign.

Most presentations imply that De Broglie waves will be the basis for what is to come, but it appears more correct to say they were the inspiration. And problems like the double slit can be worked only in one reference frame if this is all the information given. The offending wavelength which cannot be transformed as an ordinary length, even in nonrelativistic 
Galilean transformations, is associated with the superluminal phase velocity. Upon seeing De Broglie's derivation of this length from the Lorentz transform, it is obvious that it is not a physical length but a "phase length," an artifact of the Lorentz transform itself, and not something to be transformed but to be rederived in the new frame.

Traditionally, that is, for light, sound, or water waves, we think of differences in the path lengths as causing interference. In that case, if one is analyzing the frame of the particles themselves, the De Broglie wavelength is infinite and at first glance there would be no interference. How does the interference pattern remain the same for all observers, as it must, when each differently moving observer perceives a different wavelength? What we will see is that the electrons do not remain nonmoving in their own initially comoving reference frame.

\section{De Broglie's Visualization}

De Broglie's concept is still the simplest for describing free particles and is adequate for calculating interference patterns. His derivation is not widely read for two reasons. It is only the first few pages of a 73-page paper, most of which was an ad hoc attempt to apply the concept to various systems of bound particles. The following year it was superseded by the more successful Schrödinger equation, an elegant formulation suited to the bound mechanics of atoms and molecules [15]. Second, it was nearly 80 years before an English translation of De Broglie's paper appeared (Ibid. [13]).

The complex number aspect of Schrödinger's equation, its probability interpretation, and artificial zero energy point which ignores rest mass together obscure that the wavelengths are relativistic artifacts. One of the first things students learn about Schrödinger's equation is in fact that it is not applicable to relativistic motion. But even the smallest motion of a particle produces a wavelength through relativistic effects. De Broglie gave his own visualization, without a figure, described in words:

Our theorem teaches us, moreover, that this wave represents a special distribution of phase, that is to say, it is a "phase wave." To make the last point more precise, consider a mechanical comparison, perhaps a bit crude, but that speaks to one's imagination. Consider a large, horizontal circular disk, from which identical weights are suspended on springs. Let the number of such systems per unit area, i.e., their density, diminish rapidly as one moves out from the centre of the disk, so that there is a high concentration at the centre. All the weights on springs have the same period; let us set them in motion with identical amplitudes and phases. The surface passing through the centre of gravity of the weights would be a plane oscillating up and down. This ensemble of systems is a crude analogue to a parcel of energy as we imagine it to be (Ibid. [13]).

Figure 3 provides this paper's interpretation of De Broglie's "mechanical comparison," simplified still further.

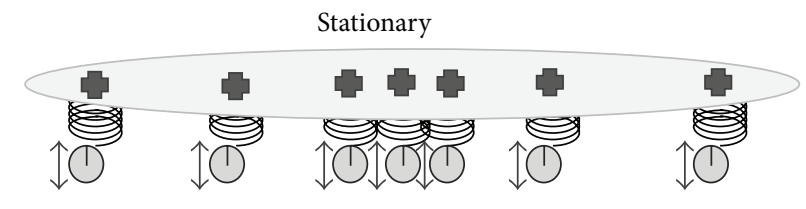

(a)

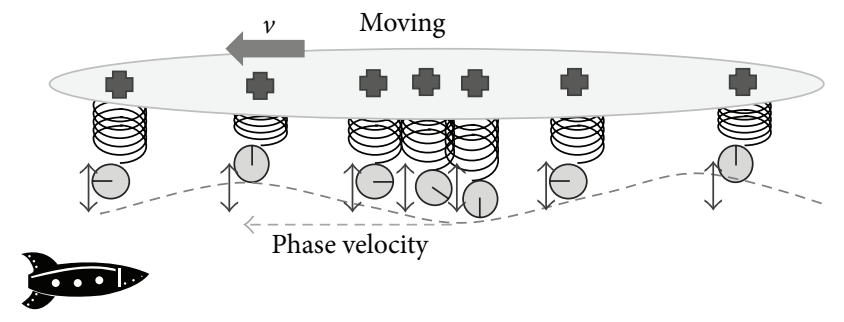

(b)

Figure 3: Stationary (a) and moving (b) De Broglie mechanical analogies.

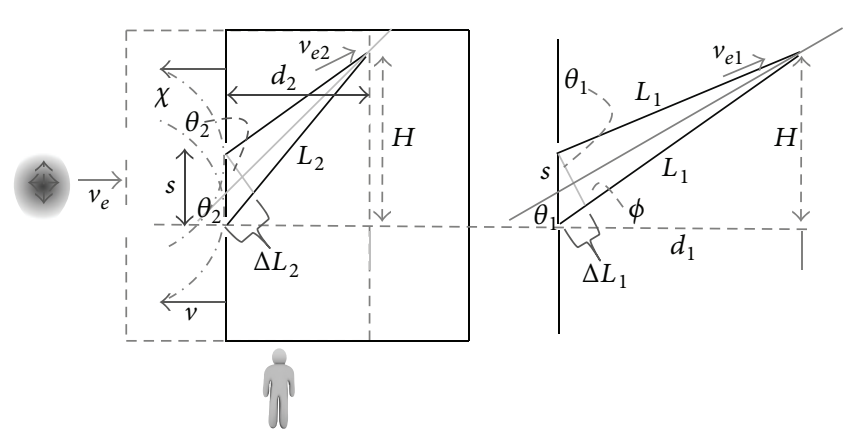

FIgURE 4: Moving double slit general setup.

In Figure 3(a), the rest frame of a particle (the large disc) is shown, and the imaginary suspended weights go up and down in phase. Each weight is shown as a clock, and, at the time-snapshot of the illustration, all clocks read the same, indicating that all points of the wave are in phase.

Figure 3(b) shows how this mechanical analogy would appear to a relatively moving inertial observer, represented by the rocket ship. In the rocket's frame, the clocks are not synchronized. Leading clocks lag, and all points do not appear to be in phase, though of course they still are in the particle's frame. See http://mclsoft.com/papers/deBroglie/ for an animated version illustrating the phase velocity (dotted wave) moving much faster than the particle.

\section{Setting up the Moving Double Slit Problem}

Consider a moving double slit approaching an electron wave packet, illustrated in Figure 4.

Velocities and distances labeled " 1 " at the right are in the frame of the slits and detector apparatus. In the frame of the observer, the slits are moving with velocity $v$ to the left. The solid lines show the position of the slit apparatus when an electron wave packet passes the slits, and the dashed lines 
show the position of the slit apparatus when that particular packet is detected. The travel path after electrons pass the slit is shortened in this reference frame, as the detection screen moves to meet them, and the velocity of the electrons is lower. The length of the path to the point of detection is labeled $L_{1}$ for the slits frame and $L_{2}$ for the observer frame. Other lengths, angles, and velocities are labeled similarly.

Notice that electron velocity is never zero if the electrons are deflected and must move vertically. Even in a frame initially comoving with the electrons only those detected at the center (not deflected) can be said to have had approximately zero velocity, and others will appear in that frame to have been scattered backward. The pair of faintly dotted semicircles $\chi$ show the scattering pattern for electron velocity $v_{e}=0$.

It is interesting that we find the notion of scattering of "particle" trajectories a necessary adjunct to understanding the "wave" property of interference, confirming again "waveparticle duality" and suggesting that student intuition about particles being "bent" as they pass slits and edges is at least half right. There are interference experiments in which only one electron at a time passes through a slit or slits, and yet the interference pattern is due to a sum over possible paths.

In the frame of the slits, where the slit velocity is zero, the electron velocities will be unchanged by scattering due to conservation of momentum. This velocity is shown as $v_{e 1}$ and determines the time $t$ between passage of the slits and detection as the electron travels along length $L_{1}$; therefore,

$$
v_{e 1}=\frac{L_{1}}{t}
$$

\section{Demonstrating Equivalence of All Frames}

It is known that phase differences are a relativistic invariant quantity, though from time to time there is discussion of it [16]. What we will do here, for pedagogical purposes, is prove that, for the case of the moving double slit, the phase difference and thus the interference pattern are the same as for a stationary slit and moving electrons. First, we will consider a nonrelativistic Galilean reference frame transformation (later to be extended), which is usual in the case of a moving apparatus in an interference experiment [17], so that the time interval $t$ is the same in all frames. The subscript " $x$ " can be either " 1 " or " 2 ":

$$
v_{e x}=\frac{L_{x}}{t}
$$

We will proceed by showing that any off-center point of maximum intensity (in phase wave arrival) at a height $H$ from center will remain the same in all frames. For convenience we assume that the slit spacing $s$ is small compared to $L$ or $H$ so that the angles $\phi$ can be treated as right angles and all other angles as either $\theta$ or $\pi / 2-\theta$. The path from the lower slit to an upper intensity maximum will be an integral multiple of wavelengths longer, shown by $\Delta L$. We do not care what the value is, only that the number of wavelengths in this interval remains the same.
Notice that for either $L$ we have

$$
L_{x}=\frac{H}{\sin \left(\theta_{x}\right)} \text {. }
$$

We can then write the De Broglie wavelength for both cases:

$$
\lambda_{x}=\frac{h}{m_{e} v_{e x}}=\left(\frac{h}{m_{e}}\right)\left(\frac{t}{L_{x}}\right)=\left(\frac{h}{m_{e}}\right)\left(\frac{t \sin \left(\theta_{x}\right)}{H}\right) .
$$

Now we find the $\Delta L$ for both cases:

$$
\Delta L_{x}=s \sin \left(\theta_{x}\right) .
$$

Finally, we investigate whether the ratio of change of $\Delta L$ is the same as the change in wavelength:

$$
\begin{aligned}
\frac{\Delta L_{2}}{\Delta L_{1}} & =\frac{s \sin \left(\theta_{2}\right)}{s \sin \left(\theta_{1}\right)}=\frac{\sin \left(\theta_{2}\right)}{\sin \left(\theta_{1}\right)}, \\
\frac{\lambda_{2}}{\lambda_{1}} & =\frac{\left(h / m_{e}\right)\left(t \sin \left(\theta_{2}\right) / H\right)}{\left(h / m_{e}\right)\left(t \sin \left(\theta_{1}\right) / H\right)}=\frac{\sin \left(\theta_{2}\right)}{\sin \left(\theta_{1}\right)} .
\end{aligned}
$$

Since (10) are the same, the exact same number of wavelengths will fit in $\Delta L$ in both cases, and we have shown that, as we knew it must, the interference pattern is the same in all frames of reference. The proof was almost trivial. The difficulty was in properly understanding how to set up the problem.

\section{The Relativistic Case}

To simplify the case of relativistic motion, a comparison will be made only between the frame of the electrons and the frame of the slits, to show that these two give the same interference pattern for highly relativistic particles. At first, we assume $H \ll d_{1}$ so that lateral dispersive velocities remain nonrelativistic in both frames, which is a constraint that we will remove later.

In the frame of the slits, for particles moving close to the speed of light, the time of transit may shift very little. In that case the primary factor in a shift in the interference pattern as the particle energy increases is that the relativistic mass increases by the Lorentz factor $\gamma$ and the wavelength decreases by that factor, constricting the entire interference pattern by $\gamma$ in the transverse direction.

In the frame of the electrons, they are not moving relativistically but the slit apparatus is, and the distance from the slits to the screen is length-contracted by the factor $\gamma$, thus reducing the travel time by that amount. To reach any given point on the screen at lateral displacement $H$, an electron would have had to travel laterally faster by the factor $\gamma$ and thus would have a lateral wavelength reduced by $\gamma$. Since this is exactly the same degree of wavelength reduction found in the frame of the slits (though not the same absolute wavelength), we conclude the interference pattern must be laterally constricted by the same amount $\gamma$.

Note that just as the Lorentz transform may be performed orthogonally in spatial direction, also the De Broglie 
wavelength can be computed independently in orthogonal directions.

Now we will remove the restriction on lateral velocity so it can be considered relativistic and introduce $\gamma_{L}$, the Lorentz factor in the lateral direction. In the original reference frame of the particles, before scattering off of the slits, the lateral wavelengths and thus the interference pattern are contracted by $\gamma_{L}$ due to mass increase. But in the reference frame of the slits, the lateral velocity is the same and there too the same lateral factor $\gamma_{L}$ applies with the same result.

Though in each case the mass of an electron appears different before scattering, we already established conformity of the interference pattern and only the relative change due to relativistic lateral motion needs be established to complete the argument.

\section{Transforming the De Broglie Frequency}

The Internet is full of speculation about some sort of "conflict" between De Broglie's concepts and Special Relativity. Most of this has to do with trying to transform the wavelength, which we have thoroughly examined. But there is also the matter of the De Broglie frequency, which being dependent on energy increases with relative velocity, rather than dilating (decreasing) as we normally think of time doing in a relatively moving reference frame.

The clocks in Figure 2(b), numbered for convenient reference, can be used to easily dismiss this concern also. Notice that the observer is using only one clock. An Einstein measurement of time in another reference frame must use at least two clocks of its own to examine a single clock in the target reference frame. This is because the changes in simultaneity with relative motion make remote measurements unreliable. If an observer's clocks are synchronized as Einstein specified, then as each of two of them passes a target clock, they can make a local measurement without simultaneity issues. For example, in the frame of the particle in Figure 2(b), clocks 2 and 3 can be compared with the observer's clock 0 as it passes, and a ratio of their delta time to the delta time from the observer's clock will show it to be running slow.

We have shown that a De Broglie wave is a wave of simultaneity in its rest frame, and so a constant phase point on that wave represents a synchronized clock time. The wave can be taken as an array of clocks. Counting the oscillations of a De Broglie wave is like the observer looking out of the window of a spaceship and reading the time from clocks in the particle frame as he passes them. This is not an Einstein 2-clocks-on-1 measurement. It is just the reverse, a 1-clockon-2 measurement. In fact, the spaceship observer's locally determined readings as he passes clocks 2 and 3 must be exactly the same numbers that the observer in the frame of the particle recorded for the exact same local events. But the spaceship observer will form the inverse of the ratio and will see time advancing more rapidly rather than dilating.

This should be stated in another way for clarity. Notice that clock 3 reads an earlier time than clock 2 in the simultaneity of the observer's reference frame. So as the observer travels from clock 3 to clock 2 , the reading reflects not only the elapsed time, but also the fixed time displacement (in the

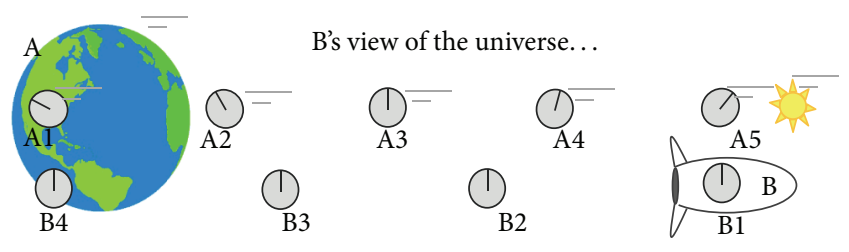

FIGURE 5: Twins paradox illustrated from traveler's viewpoint with full clock grids.

observer's frame) between these two clocks. It is like crossing time zones in a passenger jet flying east.

The 2-on-1 and 1-on-2 measurement principles can be used to explain the so-called Twins paradox [18]. Figure 5 shows the universe from B's (the traveler's) point of view. Twin A stays behind on earth. A's clock grid is skewed for B, with clocks on the left reading earlier times. If $\mathrm{B}$ uses two clocks, say $\mathrm{B} 3$ and $\mathrm{B} 4$, to measure the speed of clock $\mathrm{A} 1$ as they pass, then $B$ concludes that A's time is slower. But if $B$ uses only the $\mathrm{B} 1$ clock and compares with A1, A2, and so forth as they are passed, the progression of less-lagging clocks makes it appear that time runs faster in A's frame. The A clocks, synchronized in A's frame, give the valid current time in A's frame. If B plans to finally end up in A's frame, then to read time from the nearest A clock indeed gives the time $\mathrm{B}$ will experience if $\mathrm{B}$ stops and reenters the $\mathrm{A}$ frame at that point. This in no way limits the possible variations of simultaneity inherent in the problem. If B stops far away and A moves to catch up with B, then the final answer shifts.

The synchronized clocks in the A frame all tick at once, in the A frame. Looking at them is like looking at the De Broglie wave of a particle in the A frame, which pulses everywhere in unison in that frame. If the observer in Figure 2(b) uses two clocks and measures the De Broglie phase at a constant point in the particle's reference frame (like B using multiple clocks to measure the clock at A1), rather than a constant point in the observer frame, then the expected time dilation will be found. This is possible, but unusual, in the case of De Broglie waves. An interference pattern measures the simultaneous phase of the wave over a range of spatial points, akin to looking at all the A clocks with their accumulated skew.

De Broglie frequencies are quite high to literally count and the particle does not have a clock dial saying how many have elapsed, so it is difficult to measure a particle as it passes two lab clocks in the traditional way. One possible solution is to use a beam of coherent particles all vibrating in sync. For example, in the frame of the particle, a beam of two slightly different particle energies could be used and mixed to form a beat frequency that would function as the ticking of a clock. If this is done across a broad area, say a projection screen parallel to the relative motion, it would have the same simultaneous and nonpropagating property as the De Broglie wave of the original particle(s). It has just been made visible at a more convenient frequency by the beat frequency technique. The spaceship observer will find that if he simply looks at the nearest part of the screen, the frequency will appear higher, but if he uses multiple clocks to 
time a single point on the screen, Lorentz time dilation will be confirmed.

\section{Composite Mass}

One of the most puzzling aspects of De Broglie frequency is that though it was developed as a theory of matter waves for particles which might be thought of as a single energy packet, it is apparently a property of mass as it applies quite well to atoms and even large molecules (Ibid. [10]). Wignall has even proposed the use of De Broglie frequency as a standard of mass which requires no special mass units and suggests that quantum mechanics provides the means to demonstrate that the frequency of a bound group of particles will be proportional to the total mass (particle mass less binding energy) but is unable to cite a reference where this is actually shown [19]. So it appears that this remains an unsolved problem. In a much earlier work, Wignall hypothesizes that there may be nonlinear components of interaction of bound particles in which an additive frequency term appears and gives the analogy of amplitude radio modulation sideband frequencies [20]. For pedagogical purposes such a speculative approach is not very satisfying.

There is an obvious heuristic, however, which provides the needed frequency sum to a good approximation. We need only assume that, like Schrödinger waves, De Broglie waves are related to the probability of finding a particle. Let $p(\mathrm{~A})$ be the probability of finding $\mathrm{A}$ and $p(\mathrm{~B})$ the probability of finding $B$, and assume that these meanings continue to hold if $\mathrm{A}$ and $\mathrm{B}$ are bound together. One of the interesting aspects of De Broglie's paper (actually his thesis, which was printed in a journal) is a section treating bound particles where both are considered to be moving (Ibid. [12]). By contrast, when using Schrödinger's analysis, stationary confinement boundaries and potentials are used (which would be associated with particles, e.g., a stationary nucleus, that have infinite De Broglie wavelength). Since we are only able to find the bound pair $A B$ if we find both $A$ and $B$, then the probability of finding $A B$ must be $p(\mathrm{AB})=p(\mathrm{~A}) p(\mathrm{~B})$. If " $p$ " is a sinusoidal function, then indeed the product of two such functions reduces by a common trig identity to a term involving the sum of the frequencies of $p(\mathrm{~A})$ and $p(\mathrm{~B})$ and a term involving their difference.

The sum frequency corresponds perfectly to the frequency of the sum of the masses of A and B. The only problem is what to do with the difference frequency. Wignall's method was speculative, and we cannot use it anyway because he was not using probability, but complex valued functions. However, as an approximation we can observe two things. First, in the case of common nuclear particles, whether we treat them as hadrons (protons, neutrons), or quarks, the masses are approximately the same and the difference frequencies are therefore approximately zero. Second, in the case of the binding of electrons to a nucleus, the electron mass is to a good approximation negligible.

It would be good to also have a more thorough analysis of this effect from the standpoint of theoretical physics, and perhaps this discussion will provoke it.

\section{Demonstration of the Natural Convention of Simultaneity}

In Special Relativity, Einstein describes a convention for synchronizing remote clocks based on time-tagged communications at the speed of light and the setting of clocks based on one half the round trip travel time. In the second half of the 20th century a line of thought arose that this was not the only possible convention, and of course that is true. Reichenbach proposed a notation $t_{2}=t_{1}+\varepsilon\left(t_{3}-t_{1}\right)$, where the numbered $t$ 's are recorded times of signal transmit from $\mathrm{A}$, receive and retransmit at $\mathrm{B}$, and receive back at $\mathrm{A}$ again, and $0<\varepsilon<1$ [21]. The Einstein convention is $\varepsilon=1 / 2$. Several investigators have pointed out that such things as the time difference in the aging of twins in a complete two-way voyage do not depend on the convention of simultaneity, and for those purposes it is "just a convention" [22]. The one-way description of relative clock rates is of course dependent on it.

Short of measuring the one-way speed of light, which is very problematic, there is a phenomenon which can be measured to demonstrate what convention is used, at least in one case, by nature. The De Broglie wavelength, as seen in Figures 2(b) and 3(b), is directly associated with clock-like oscillations that define the phase of the wave, and the length of the wave is a direct result of the Lorentz transform, which implements the Einstein convention. For another convention, De Broglie wavelengths would be different, and maxima and minima in interference patterns would fall at different places. This is about as likely as that they would fall at different places because the double slit is moving instead of the electrons, in other words, not likely at all. So for De Broglie waves, and we suspect therefore for quantum systems generally, the Einstein convention is natural. Not only is it natural, but also it is automatic. Swann pointed out in 1960 that whereas macroscopic clocks had to be manually resynchronized after acceleration, quantum systems automatically come into synchronization as Einstein reference frames [23].

\section{Conclusion}

Retracing De Broglie's derivation of the phase velocity and associated wave, with visualizations, gives important insight into the meaning of De Broglie waves that will help students analyze and set up problems and avoid traps like trying to do Lorentz transforms of the De Broglie wavelength. Certainly we would expect to see fewer papers claiming to have rediscovered the relation between De Broglie waves and the Lorentz transform or deriving the Lorentz transform from De Broglie waves (circular). There is an opportunity, if desired, to demystify the variation of the De Broglie frequency which increases rather than dilates with motion. The particle always vibrates at the same rate in its own frame like any other clock.

Continuing on to analyze the moving double slit does two useful things. It provides continuity with Special Relativity, avoiding the impression that there is anything special about the frame of the measurement apparatus. And it guides the students as to how to set up a variety of related interference problems. 
More advanced students may be interested in the heuristic approach to understanding how the frequency of a sum of masses is the sum of frequencies, and perhaps one of them will eventually produce Wignall's hoped-for satisfactorily complete theoretical analysis. For those going on to specialize in relativity, understanding that De Broglie waves particularly and quantum systems generally follow the laws of relativity automatically, whereas macroscopic clocks might require adjustment after acceleration, may provide a basis for future insight into the nature of space-time.

\section{Conflict of Interests}

The author declares that there is no conflict of interests regarding the publication of this paper.

\section{References}

[1] B. S. Ambrose, P. S. Shaffer, R. N. Steinberg, and L. C. McDermott, "An investigation of student understanding of single-slit diffraction and double-slit interference," American Journal of Physics, vol. 67, no. 2, pp. 146-155, 1999.

[2] R. Martins, "Louis de Broglie's Struggle with the Wave-Particle Dualism, 1923-1925," in HQ-1 Conference, Quantum History Project, Jointly: Fritz Haber Institute, Max Planck Society, Max Planck Institute for the History of Science, 2007.

[3] H. Vries, "The simplest, and the full derivation of Magnetism as a Relativistic side effect of ElectroStatics," in Understanding Relativistic Quantum Field Theory, Physics Quest, 2008, http://www.chip-architect.com/physics/.

[4] E. M. Purcell, Electricity and Magnetism, vol. 2 of Berkeley Physics Course, McGraw Hill, 2nd edition, 1984.

[5] T. Mattson, "Lorentz transformation of the de broglie relation," Physics Forums, Article ID 76060, 2005.

[6] W. E. Baylls, "De Broglie waves as an effect of clock desynchronization," Canadian Journal of Physics, vol. 85, no. 12, pp. 13171323, 2007.

[7] R. Dogra, "A new proposal combining quantum mechanics and the special theory of relativity," Apeiron, vol. 9, no. 2, 2002.

[8] H. Ylmaz, "Lorentz transformations and wave-particle unity," Physics Essays, vol. 23, no. 2, pp. 334-336, 2010.

[9] C. Davisson and L. H. Germer, "Diffraction of electrons by a crystal of nickel," Physical Review, vol. 30, no. 6, pp. 705-741, 1927.

[10] O. Nairz, M. Arndt, and A. Zeilinger, "Quantum interference experiments with large molecules," American Journal of Physics, vol. 71, no. 4, pp. 319-325, 2003.

[11] C. Bruce, "Is de Broglie matter wave a mass or a particle hypothesis?” Physics Stack Exchange, 213677, March 2015, http://physics.stackexchange.com/questions/173696/is-de-broglie-matter-wave-a-mass-or-a-particle-hypothesis.

[12] L. De Broglie, “The wave nature of the electron," Nobel Lecture, 1929.

[13] L. De Broglie, "Recherches sur la théorie des quanta," Annales de Physique, vol. 10, no. 3, p. 22, 1925 (French), translated by A. F. Kracklauer.

[14] B. H. Bransden and C. J. Joachain, Quantum Mechanics, Pearson Education Ltd, Edinburg Gate, 2nd edition, 2000.

[15] E. Schrödinger, "An undulatory theory of the mechanics of atoms and molecules," Physical Review, vol. 28, no. 6, pp. 10491070, 1926.
[16] A. Gjurchinovski, "Is the phase of plane waves a frameindependent quantity? Comment on 'the invariance of the phase of waves among inertial frames is questionable' by Huang Young-Sea," Europhysics Letters, vol. 83, no. 1, Article ID 10001, 2008.

[17] A. G. Klein, G. I. Opat, A. Cimmino, A. Zeilinger, W. Treimer, and R. Gähler, "Neutron propagation in moving matter: the Fizeau experiment with massive particles," Physical Review Letters, vol. 46, no. 24, pp. 1551-1554, 1981.

[18] R. L. Shuler, "The twins-clock paradox history and perspectives," Journal of Modern Physics, vol. 05, no. 12, pp. 1062-1078, 2014.

[19] J. W. G. Wignall, "Some comments on the definition of mass," Metrologia, vol. 44, no. 3, pp. L19-L22, 2007.

[20] J. W. G. Wignall, "De Broglie waves and the nature of mass," Foundations of Physics, vol. 15, no. 2, pp. 207-227, 1985.

[21] H. Reichenbach, The Philosophy of Space \& Time, Dover Publications, New York, NY, USA, 1958.

[22] T. A. Debs and M. L. G. Redhead, “The twin 'paradox' and the conventionality of simultaneity," American Journal of Physics, vol. 64, no. 4, pp. 384-392, 1996.

[23] W. F. G. Swann, "Certain matters in relation to the restricted theory of relativity, with special reference to the clock paradox and the paradox of the identical twins. I. Fundamentals," American Journal of Physics, vol. 28, no. 55, pp. 55-64, 1960. 

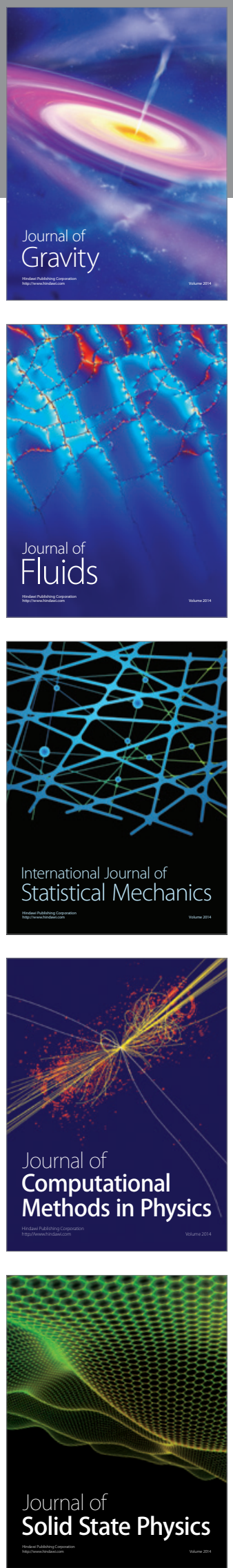

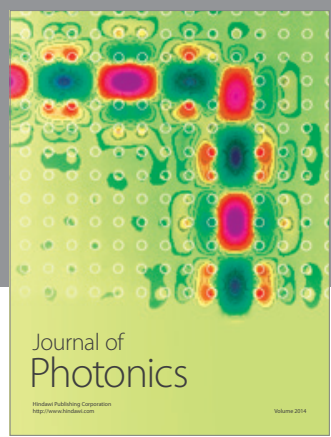

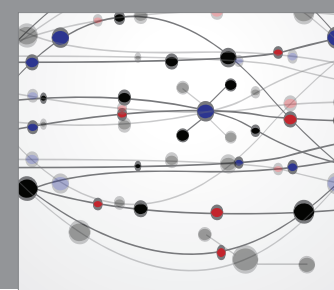

The Scientific World Journal

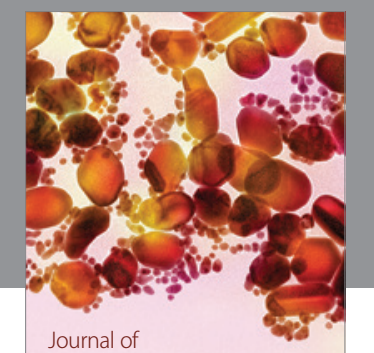

Soft Matter
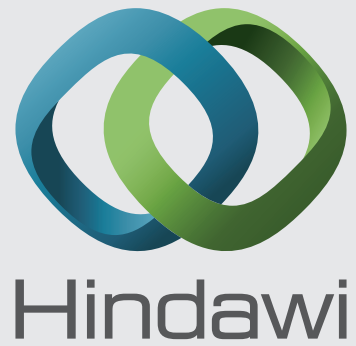

Submit your manuscripts at

http://www.hindawi.com
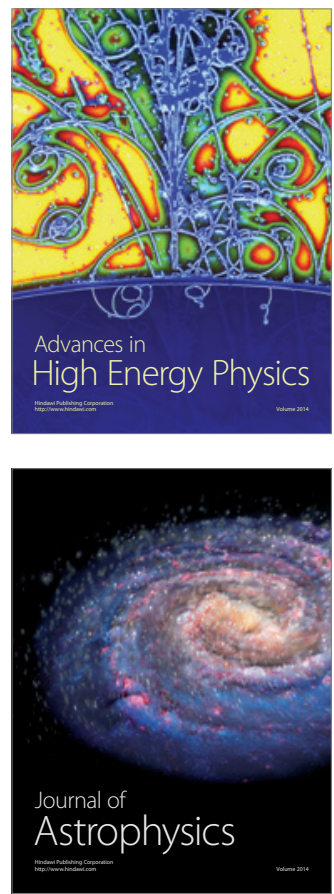
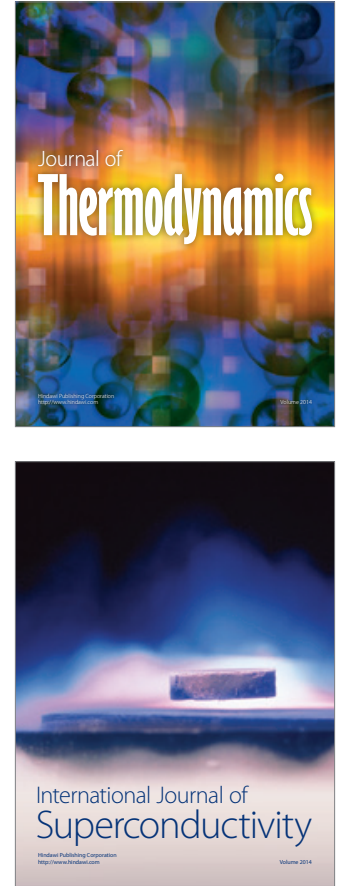
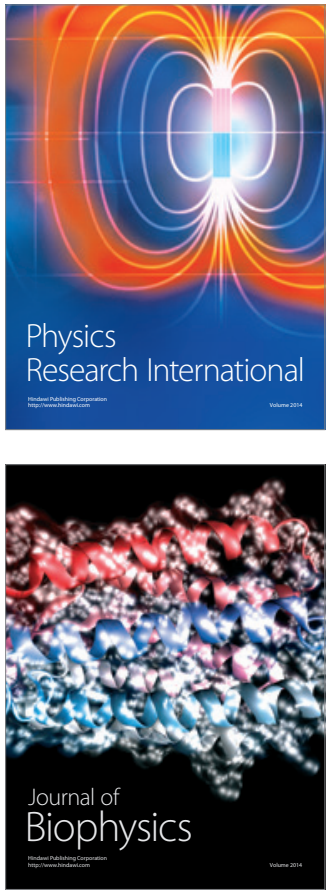
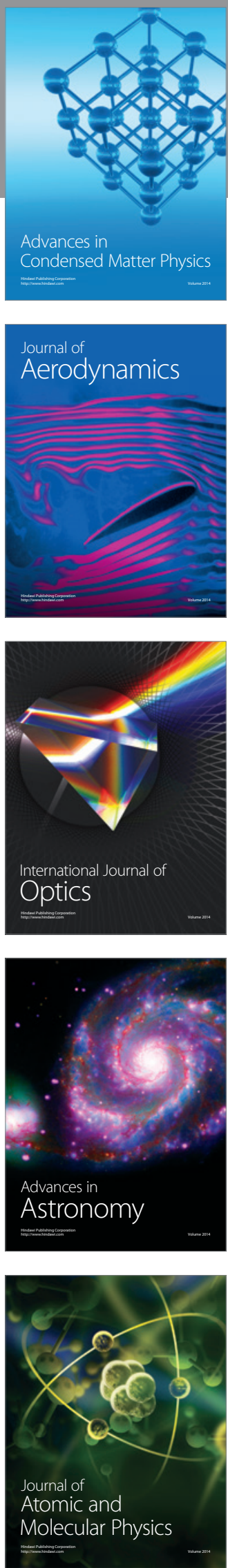